\title{
Dry-matter intake constraints on production in dual-purpose goats: a possible solution by feeding defoliated maize leaves
}

\author{
P. P. Semenye \\ SR-CRSP ARI, Winrock International, PO Box 252, Maseno, Kenya
}

\section{Material and methods}

Feeding trials were conducted at Maseno, Kenya, based on maize leaves taken from the plant (defoliated) from three plots measuring 0.25 ha each. On average each plot produced $359 \pm 40 \mathrm{~kg}$ dry matter of defoliated leaves. Defoliation which commenced at the tasselling stage at the rate of one leaf per week per maize plant did not affect the grain yield. The defoliated maize leaves forage had $120 \mathrm{~g}$ crude protein per $\mathrm{kg}$ and in vivo dry matter digestibility of 0.6 .
Leaves from the first plot were assigned to two lactating does. For 3 months the two does were fed only with leaves from this plot. Within this period each doe produced $81 \mathrm{~kg}$ milk. The second plot served as a control with no removal of leaves. The third plot sustained growth of three yearlings for 3 months at the rate of $45 \mathrm{~g} /$ day per yearling. This performance was compared with two other pens of yearlings given a grass mixture or concentrates.

\section{Results}

Table 1 Maize grain and defoliated leaf production per 0.25 ha

\begin{tabular}{|c|c|c|c|c|c|}
\hline & \multirow{2}{*}{$\begin{array}{l}\text { Plot } 1 \\
\text { (Does) }\end{array}$} & \multirow{2}{*}{$\begin{array}{c}\text { Plot } 2 \\
\text { (Control) }\end{array}$} & \multirow{2}{*}{$\begin{array}{c}\text { Plot } 3 \\
\text { (Yearlings) }\end{array}$} & \multicolumn{2}{|c|}{ Overall } \\
\hline & & & & Mean & s.d. \\
\hline No. of plants & 7875 & 7536 & 9278 & 8230 & 924 \\
\hline No. of leaves & 110250 & 105504 & 129892 & 115215 & 12930 \\
\hline Leaf dry matter (kg) & 344 & 329 & 405 & 359 & 40 \\
\hline Grain yield (kg) & 1362 & 1263 & 1387 & 1337 & 66 \\
\hline Grain yield per plant (g) & 173 & 168 & 149 & 163 & 13 \\
\hline
\end{tabular}

Table 2 Ch'mical composition of the diets $(\mathrm{g} / \mathrm{kg}$ )

\begin{tabular}{|c|c|c|c|c|c|c|}
\hline & \multicolumn{2}{|c|}{ Grass mixture } & \multicolumn{2}{|c|}{ Maize leaves } & \multicolumn{2}{|c|}{ Concentrates } \\
\hline & Mean & s.d. & Mean & s.d. & Mean & s.d. \\
\hline Dry matter (DM) & $310^{a}$ & 32 & $340^{n}$ & 36 & $940^{b}$ & 9 \\
\hline Crude protein & $120^{\circ}$ & 8 & $120^{a}$ & 8 & $180^{b}$ & 10 \\
\hline In vitro DM digestibility & $0.57^{\mathrm{a}}$ & 0.021 & $0.64^{b}$ & 0.018 & 0.78 & 0.019 \\
\hline In zivo DM digestibility & $0.61^{a}$ & 0.064 & $0.60^{a}$ & 0.045 & $0.68^{b}$ & 0.029 \\
\hline
\end{tabular}

akx Means in the same row having no superscript in common differ at the $P<0.05$ level. 
Table 3 Performance of dual-purpose goat yearlings fed grass mixture, defoliated maize leaves or concentrates

\begin{tabular}{lccr}
\hline \hline & Grass mixture & Maize leaves & Concentrates \\
\hline Dry matter intake (g/day) & 764 & 788 & 723 \\
Dry matter intake (g/kg body weight) & 35 & 35 & 31 \\
Average daily gain (g/day) & 27 & 45 & 89 \\
\hline \hline
\end{tabular}

Table 4 Production of defoliated maize leaves per plot size and dual-purpose goat feeding days

\begin{tabular}{ccccc}
\hline \hline & & Defoliated leaves \\
Maize plot & Droduction \\
size (ha) & potential (no.) & yield & $\begin{array}{c}\text { Potential feeding days } \\
\text { for a 40-kg doe producing } \\
0.7 \mathrm{~kg} \text { milk per day }\end{array}$ \\
\cline { 3 - 5 } & 4.0 & 1437 & per season & per yeart \\
0.5 & 230430 & 719 & 1105 & 2210 \\
0.25 & 115215 & 359 & 553 & 1106 \\
0.1 & 46086 & 144 & 276 & 552 \\
\hline \hline
\end{tabular}

+ Two growing seasons per year.

\section{Discussion}

In maize-growing areas, where there is no pasture or land for growing fodder, it is recommended that the feeding and management of goats should be matched to maize production. Lactation period should coincide with availability of maize leaves, while yearlings should take full advantage of maize leaves in the diet because it is likely to give a better performance than a grass-mixture diet. 